\title{
Input-to-state stability analysis for interconnected difference equations with delay
}

\author{
Rob H. Gielen · Mircea Lazar • Andrew R. Teel
}

Received: 6 May 2011 / Accepted: 16 February 2012 / Published online: 4 March 2012

(C) The Author(s) 2012. This article is published with open access at Springerlink.com

\begin{abstract}
Input-to-state stability (ISS) of interconnected systems with each subsystem described by a difference equation subject to an external disturbance is considered. Furthermore, special attention is given to time delay, which gives rise to two relevant problems: (i) ISS of interconnected systems with interconnection delays, which arise in the paths connecting the subsystems, and (ii) ISS of interconnected systems with local delays, which arise in the dynamics of the subsystems. The fact that a difference equation with delay is equivalent to an interconnected system without delay is the crux of the proposed framework. Based on this fact and small-gain arguments, it is demonstrated that interconnection delays do not affect the stability of an interconnected system if a delay-independent small-gain condition holds. Furthermore, also using small-gain arguments, ISS for interconnected systems with local delays is established via the Razumikhin method as well as the Krasovskii approach. A combination of the results for interconnected systems with interconnection delays and local delays, respectively, provides a framework for ISS analysis of general interconnected systems with delay. Thus, a scalable ISS analysis method is obtained for large-scale interconnections of difference equations with delay.
\end{abstract}

This paper was partially presented at the 18th IFAC World Congress, Milano, Italy, 2011.

R. H. Gielen $(\varangle) \cdot$ M. Lazar

Electrical Engineering Department, Eindhoven University of Technology,

Eindhoven, The Netherlands

e-mail: r.h.gielen@tue.nl

M. Lazar

e-mail:m.lazar@tue.nl

A. R. Teel

Electrical and Computer Engineering Department, University of California,

Santa Barbara, USA

e-mail: teel@ece.ucsb.edu 
Keywords Large-scale systems · Time delay · Difference equations · Lyapunov methods $\cdot$ Small-gain theorem

\section{Introduction}

Large-scale interconnections of dynamical systems, such as power systems, chemical processes and urban water supply networks, form an important topic within the field of control systems, see, e.g., $[21,30,31]$ and the references therein. The stability analysis, e.g., based on Lyapunov theory, of such systems is generally hampered by the large size and complexity of the overall system. Therefore, a stability analysis of the subsystems is typically performed first. Then, the stability of the interconnected system is studied. To this end, small-gain theorems, such as the ones presented in [2] and [12], can be used. Alternatively, vector Lyapunov functions [16] or dissipativity theory [33] can also be used for the stability analysis of interconnected systems.

In practice, interconnections of dynamical systems, such as, for example, power systems, often show a geographical separation of the subsystems. Hence, the propagation of signals takes place over large distances which can induce interconnection delays. Furthermore, due to inherent delays in the dynamical processes, local delays can also arise in the subsystems. Indeed, for example, in power systems interconnection delays are introduced by water flowing through rivers that connect hydro-thermal power plants $[22,32]$ while local delays can be introduced by human operators in the local control loops. As delays can cause the instability of a dynamical system [5,13], any stability analysis framework must take delays into account. For example, the stability analysis of a single system with delay can, among others, be performed using the Razumikhin method or the Krasovskii approach, see, e.g., [5,13] and [4,6,18] for continuous- and discrete-time systems, respectively. In fact, the Razumikhin method is [27] a type of small-gain approach for systems with delay and the Krasovskii approach can be considered as a standard Lyapunov approach adapted to systems with delay.

Recently, based on the aforementioned extensions of Lyapunov theory, several small-gain theorems for interconnected systems with delay were proposed. For example, based on the Krasovskii approach, the integral input-to-state stability (ISS) analysis for interconnected systems with both interconnection and local delays was performed in [8,9]. A similar result was obtained in the context of ISS in [1]. Furthermore, also in [1], it was indicated that ISS can also be established based on the Razumikhin method and small-gain arguments. Alternatively, the relation of the Razumikhin method to the small-gain theorem given in [27] was used in [28] to formulate a small-gain theorem for interconnected systems with both interconnection and local delays. A different approach was taken in [29], where a small-gain theorem for interconnected systems with both interconnection and local delays was established using standard small-gain arguments, but without using Lyapunov theory. However, none of the above results applies to interconnections of difference equations with delay. Moreover, interconnection delays and local delays have thus far mostly been considered at the same time, while in [8] it was shown that considering them separately can be advantageous. Indeed, as it will also be shown in this paper, interconnection 
delays, in contrast to local delays, do not affect the stability of the overall system if a delay-independent small-gain condition holds.

Therefore, in this paper, ISS for interconnections of difference equations with delay is studied. Moreover, interconnection delays and local delays are considered separately. Before considering interconnected systems with delay, ISS for a single system with delay will be studied to obtain techniques that can be applied to interconnected systems thereafter. To this end, a transformation of a system with delay into an interconnected system without delay, by defining each delayed state as a new subsystem, is developed. Thus, ISS of a system with delay can be studied using the small-gain theorem for interconnected systems. As a consequence, it is shown that the Razumikhin method is an exact application of the small-gain theorem to systems with delay. As a by-product of this insight, a method to construct a Lyapunov-Krasovskii function (LKF) from a Lyapunov-Razumikhin function (LRF) is also obtained. Then, interconnected systems with delay are considered. Based on the transformation indicated above and small-gain arguments, it is demonstrated that interconnection delays do not affect the stability of an interconnected system if a delay-independent small-gain condition holds. Furthermore, under a similar small-gain condition, it is shown that interconnected systems with local delays admit a LRF for the interconnected system if each subsystem admits a LRF. Similarly, it is shown that the interconnected system admits a LKF if each subsystem admits a LKF and a small-gain condition is satisfied. A combination of the results for interconnected systems with interconnection delays and local delays, respectively, provides a scalable framework for ISS analysis of general interconnected systems with delay.

The remainder of the paper is organized as follows. Section 2 provides some useful preliminaries. Then, Section 3 discusses the relation between the small-gain theorem and the Razumikhin method. Next, Sect. 4 studies ISS for interconnected systems with interconnection delays. Thereafter, in Sect. 5, interconnected systems with local delays are considered and Sect. 6 presents the results for general interconnected systems with delay. Finally, the conclusions are drawn in Sect. 7 while the Appendix contains the proof of a fundamental theorem.

\section{Preliminaries}

Necessary notation, basic definitions and a suitable notion of stability will be introduced in this section.

\subsection{Notation and basic definitions}

Let $\mathbb{R}, \mathbb{R}_{+}, \mathbb{Z}$ and $\mathbb{Z}_{+}$denote the field of real numbers, the set of non-negative reals, the set of integers and the set of non-negative integers, respectively. For every $c \in$ $\mathbb{R}$ and $\Pi \subseteq \mathbb{R}$, define $\Pi_{\geq c}:=\{k \in \Pi \mid k \geq c\}$ and similarly $\Pi_{\leq c}$. Furthermore, $\mathbb{R}_{\Pi}:=\mathbb{R} \cap \Pi$ and $\overline{\mathbb{Z}}_{\Pi}:=\mathbb{Z} \cap \Pi$. For a vector $x \in \mathbb{R}^{n}$, let $[x]_{i}, i \in \mathbb{Z}_{[1, n]}$, denote the $i$ th component of $x$ and let $\|x\|$ denote an arbitrary norm. For a matrix $A \in \mathbb{R}^{n \times n}$ let $[A]_{i, j}, i, j \in \mathbb{Z}_{[1, n]}$, denote the $i j$ th entry of $A$. Let $\mathbf{x}:=\{x(l)\}_{l \in \mathbb{Z}_{+}}$ with $x(l) \in \mathbb{R}^{n}$ for all $l \in \mathbb{Z}_{+}$denote an arbitrary sequence and define $\|\mathbf{x}\|:=$ 
$\sup \left\{\|x(l)\| \mid l \in \mathbb{Z}_{+}\right\}$. Furthermore, $\mathbf{x}_{\left[c_{1}, c_{2}\right]}:=\{x(l)\}_{l \in \mathbb{Z}_{\left[c_{1}, c_{2}\right]}}$, with $c_{1}, c_{2} \in \mathbb{Z}$, denotes a sequence that is ordered monotonically with respect to the index $l \in \mathbb{Z}_{\left[c_{1}, c_{2}\right]}$.

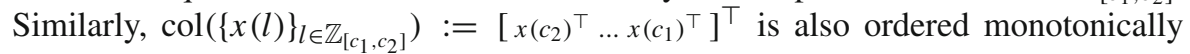
(albeit in a decreasing fashion from top to bottom) with respect to the index $l$. Let $\mathbb{S}^{h}:=\mathbb{S} \times \cdots \times \mathbb{S}$ for any $h \in \mathbb{Z}_{\geq 1}$ denote the $h$-times cross-product of an arbitrary set $\mathbb{S} \subseteq \mathbb{R}^{n}$. Let Id $: \mathbb{R} \rightarrow \mathbb{R}$ denote the identity function. For two functions $\varphi_{1}: \mathbb{R}^{n} \rightarrow \mathbb{R}^{m}$ and $\varphi_{2}: \mathbb{R}^{l} \rightarrow \mathbb{R}^{n}$, let $\varphi_{1} \circ \varphi_{2}(x):=\varphi_{1}\left(\varphi_{2}(x)\right)$ for all $x \in \mathbb{R}^{l}$. Let $\varphi: \mathbb{R}_{+} \rightarrow \mathbb{R}_{+}$. Define $\varphi^{k}(s):=\varphi \circ \varphi^{k-1}(s)$ for all $k \in \mathbb{Z}_{\geq 1}$ and all $s \in \mathbb{R}_{+}$, where $\varphi^{0}(s):=s$. Furthermore, $\varphi \in \mathscr{K}$ if it is continuous, strictly increasing and $\varphi(0)=0 . \varphi \in \mathscr{K}_{\infty}$ if $\varphi \in \mathscr{K}$ and $\lim _{s \rightarrow \infty} \varphi(s)=\infty$. The notation $\varphi \in \mathscr{K} \cup\{0\}$ $\left(\varphi \in \mathscr{K}_{\infty} \cup\{0\}\right)$ is used to denote that either $\varphi \in \mathscr{K}\left(\varphi \in \mathscr{K}_{\infty}\right)$ or $\varphi(s)=0$ for all $s \in \mathbb{R}_{+}$. Let $\beta: \mathbb{R}_{+} \times \mathbb{R}_{+} \rightarrow \mathbb{R}_{+} \cdot \beta \in \mathscr{K} \mathscr{L}$ if for each fixed $s \in \mathbb{R}_{+}, \beta(\cdot, s) \in \mathscr{K}$ and for each fixed $r \in \mathbb{R}_{+}, \beta(r, \cdot)$ is decreasing and $\lim _{s \rightarrow \infty} \beta(r, s)=0$.

\subsection{Delay difference equations}

Consider systems described by the delay difference equation (DDE)

$$
x(k+1)=F\left(\mathbf{x}_{[k-h, k]}, u(k)\right), \quad k \in \mathbb{Z}_{+},
$$

where $\mathbf{x}_{[k-h, k]} \in\left(\mathbb{R}^{n}\right)^{h+1}$ is a sequence of (delayed) states, $h \in \mathbb{Z}_{+}$is the maximal delay and $u(k) \in \mathbb{R}^{m}$ is a disturbance input. Furthermore, $F:\left(\mathbb{R}^{n}\right)^{h+1} \times \mathbb{R}^{m} \rightarrow \mathbb{R}^{n}$ is a function that admits the origin as fixed point, i.e., $F\left(\mathbf{0}_{[k-h, k]}, 0\right)=0$. The notation $\left\{x\left(k, \mathbf{x}_{[-h, 0]}, \mathbf{u}_{[0, k-1]}\right)\right\}_{k \in \mathbb{Z}_{>1}}$ is used to denote a trajectory of the system (1) from initial condition $\mathbf{x}_{[-h, 0]} \in\left(\mathbb{R}^{n}\right)^{h+1}$ with disturbance $\mathbf{u}_{[0, k-1]}:=\{u(i)\}_{i \in \mathbb{Z}_{[0, k-1]}}, u(i) \in \mathbb{R}^{m}$.

Definition 1 The DDE (1) is called input-to-state stable (ISS) if there exist a $\beta \in$ $\mathscr{K} \mathscr{L}$ and a $\gamma_{u} \in \mathscr{K}$, such that for all $k \in \mathbb{Z}_{\geq 1}$ it holds that

$$
\left\|x\left(k, \mathbf{x}_{[-h, 0]}, \mathbf{u}_{[0, k-1]}\right)\right\| \leq \max \left\{\beta\left(\left\|\mathbf{x}_{[-h, 0]}\right\|, k\right), \gamma_{u}\left(\left\|\mathbf{u}_{[0, k-1]}\right\|\right)\right\},
$$

for all $\mathbf{x}_{[-h, 0]} \in\left(\mathbb{R}^{n}\right)^{h+1}$ and all $\mathbf{u}_{[0, k-1]} \in\left(\mathbb{R}^{m}\right)^{k}$.

Note that, ISS, cf. Definition 1, is a global property which is often referred to as global ISS. Furthermore, as $\max \{r, s\} \leq r+s$ and $r+s \leq \max \{2 r, 2 s\}$ for all $r, s \in \mathbb{R}_{+}$, Definition 1 is equivalent to Definition 2.2 in [18], which was also indicated therein.

\subsection{A small-gain theorem for interconnected systems}

Consider an interconnection of $N \in \mathbb{Z}_{\geq 2}$ subsystems. The dynamics of the $i$ th subsystem, $i \in \mathbb{Z}_{[1, N]}$, is described by a difference equation, i.e.,

$$
x_{i}(k+1)=g_{i}\left(x_{1}(k), \ldots, x_{N}(k), u(k)\right), \quad k \in \mathbb{Z}_{+},
$$

where $x_{i}(k) \in \mathbb{R}^{n_{i}}, u(k) \in \mathbb{R}^{m}$ is the input to the subsystem and $g_{i}: \mathbb{R}^{n_{1}} \times \cdots \times$ $\mathbb{R}^{n_{N}} \times \mathbb{R}^{m} \rightarrow \mathbb{R}^{n_{i}}, i \in \mathbb{Z}_{[1, N]}$, is a function that admits the origin as fixed point. Note 
that it can be assumed without any restriction to the generality of the results that all subsystems have the same disturbance input. To describe the complete interconnected system, let $x:=\operatorname{col}\left(\left\{x_{i}\right\}_{i \in \mathbb{Z}_{[1, N]}}\right) \in \mathbb{R}^{n}$, which yields

$$
x(k+1)=G(x(k), u(k)), \quad k \in \mathbb{Z}_{+},
$$

where $n:=\sum_{i=1}^{N} n_{i}$ and $G: \mathbb{R}^{n} \times \mathbb{R}^{m} \rightarrow \mathbb{R}^{n}$ is obtained from the functions $g_{i}$, $i \in \mathbb{Z}_{[1, N]}$, i.e., $G(x, u)=\operatorname{col}\left(\left\{g_{i}\left(x_{1}, \ldots, x_{N}, u\right)\right\}_{i \in \mathbb{Z}_{[1, N]}}\right)$.

Next, let $\alpha_{1, i}, \alpha_{2, i} \in \mathscr{K}_{\infty}, \mu_{i} \in \mathscr{K} \cup\{0\}$, for all $i \in \mathbb{Z}_{[1, N]}$, and let $\gamma_{i, j} \in \mathscr{K}_{\infty} \cup\{0\}$ for all $i, j \in \mathbb{Z}_{[1, N]}$. Then, consider the functions $W_{j}: \mathbb{R}^{n_{j}} \rightarrow \mathbb{R}_{+}, j \in \mathbb{Z}_{[1, N]}$, that satisfy

$$
\alpha_{1, j}\left(\left\|x_{j}\right\|\right) \leq W_{j}\left(x_{j}\right) \leq \alpha_{2, j}\left(\left\|x_{j}\right\|\right), \quad \forall x_{j} \in \mathbb{R}^{n_{j}} .
$$

Definition 2 Let $\gamma_{i, i}(s)<s$ for all $s \in \mathbb{R}_{>0}$. A function $W_{i}, i \in \mathbb{Z}_{[1, N]}$, that satisfies

$$
W_{i}\left(g_{i}\left(x_{1}, \ldots, x_{N}, u\right)\right) \leq \max \left\{\max _{j \in \mathbb{Z}_{[1, N]}} \gamma_{i, j} \circ W_{j}\left(x_{j}\right), \mu_{i}(\|u\|)\right\},
$$

for all $x_{j} \in \mathbb{R}^{n_{j}}, j \in \mathbb{Z}_{[1, N]}$, and all $u \in \mathbb{R}^{m}$ is called an ISS-Lyapunov function (ISS-LF) for subsystem (2).

Note that Definition 2 requires that $W_{i}$ is a standard ISS-LF (cf. Definition 3.2 in [10]) for subsystem (2) with respect to the input $u$, i.e., for $x_{j}=0$ for all $j \neq i$. Moreover, it also requires that, if there is a connection from the $j$ th subsystem to the $i$ th subsystem, the influence of the state $x_{j}$ on (2) can be bounded via $\gamma_{i, j}$.

Next, a lemma, which is fundamental for the derivation of many results in this paper, is recalled. Moreover, based on [2, Corollary 5.7], a result for continuous-time systems, and using Definition 2, a nonlinear small-gain theorem for interconnections of difference equations is established.

Lemma 1 Consider the functions $\gamma_{i, j}$. If, for all $y \in \mathbb{R}_{+}^{N} \backslash\{0\}$, there exists a $i(y) \in$ $\mathbb{Z}_{[1, N]}$ such that $\max _{j \in \mathbb{Z}_{[1, N]}} \gamma_{i, j}\left([y]_{j}\right)<[y]_{i}$, then there exist $\sigma_{i} \in \mathscr{K}_{\infty}, i \in \mathbb{Z}_{[1, N]}$, such that $\max _{j \in \mathbb{Z}_{[1, N]}} \gamma_{i, j} \circ \sigma_{j}\left([y]_{j}\right)<\sigma_{i}\left([y]_{i}\right)$ for all $y \in \mathbb{R}_{+}^{N} \backslash\{0\}$ and all $i \in \mathbb{Z}_{[1, N]}$.

Lemma 1 appeared as claim (iii) of Theorem 5.2 in [2].

Theorem 1 Suppose that all subsystems (2), $i \in \mathbb{Z}_{[1, N]}$, admit an ISS-LF. Furthermore, suppose that for all $y \in \mathbb{R}_{+}^{N} \backslash\{0\}$, there exists a $i(y) \in \mathbb{Z}_{[1, N]}$ such that

$$
\max _{j \in \mathbb{Z}_{[1, N]}} \gamma_{i, j}\left([y]_{j}\right)<[y]_{i}
$$

Then, the following claims hold:

(i) There exist $\sigma_{i} \in \mathscr{K}_{\infty}, i \in \mathbb{Z}_{[1, N]}$, such that

$$
W(x)=\max _{i \in \mathbb{Z}_{[1, N]}} \sigma_{i}^{-1}\left(W_{i}\left(x_{i}\right)\right)
$$

is an ISS-LF for the interconnected system (3);

(ii) The interconnected system (3) is ISS. 
The proof of Theorem 1 can be found in Appendix A. Note that an explicit expression for the functions $\sigma_{i}$ can be obtained using the ideas presented in [12]. Therefore, Theorem 1 is constructive.

Alternative small-gain theorems for interconnections of difference equations, which also parallel the continuous-time results in [2], can be found in, e.g., [11,15,20,24]. Techniques that provide a more flexible framework, e.g., based on vector Lyapunov functions, see [12], are not pursued here. This is motivated by the fact that involving functions that depend on the complete state vector is less likely to yield a scalable ISS analysis method, which is the main objective of this work.

Remark 1 Definition 2, and several similar definitions below, can be relaxed in the sense that ${ }^{1} \gamma_{i, j} \in \mathscr{G}$ instead of $\gamma_{i, j} \in \mathscr{K}_{\infty} \cup\{0\}$. Indeed, suppose that $W_{i}: \mathbb{R}^{n_{i}} \rightarrow \mathbb{R}_{+}$ and $\tilde{\gamma}_{i, i} \in \mathscr{G}$ satisfy the hypotheses of Definition 2. Let $\gamma_{i, i}(s)=\frac{\tilde{\gamma}_{i, i}(s)+s}{2}$, for all $s \in \mathbb{R}_{+}$. Then, $\gamma_{i, i} \in \mathscr{K}_{\infty}$ and $\tilde{\gamma}_{i, i}(s) \leq \gamma_{i, i}(s)<s$ for all $s \in \mathbb{R}_{>0}$. Therefore, $W_{i}$ and $\gamma_{i, i}$ also satisfy the hypotheses of Definition 2. For simplicity, we chose to restrict ourselves to $\gamma_{i, j} \in \mathscr{K}_{\infty} \cup\{0\}$. In fact, generalizing the results in this paper to $\gamma_{i, j} \in \mathscr{G}$ relies mainly on the extension of Lemma 1 to $\gamma_{i, j} \in \mathscr{G}$.

Remark 2 The results in this paper allow, under suitable assumptions, for a straightforward generalization from difference equations to difference inclusions. To this end, the Lyapunov decrease condition, as in, e.g., Definition 2, should be required to hold for all states in the set-valued map which dictates the state update. However, for simplicity, all results in this paper are presented for difference equations.

In what follows, (the techniques used to prove) Theorem 1 will be used to obtain small-gain theorems for interconnections of difference equations with interconnection delays and/or local delays.

\section{ISS analysis for delay difference equations}

Before considering interconnected systems with delay, ISS for a single system with delay will be studied to obtain techniques that can be applied to interconnected systems thereafter. Therefore, in this section, ISS for the DDE (1) is studied. To this end, a transformation of (1) into an interconnection of difference equations without delay will be developed such that Theorem 1 can be applied. Thus, it will be established that the Razumikhin method is an exact application of the small-gain theorem.

Each delayed state of the DDE (1) can be considered as one of the subsystems (2). Thus, an interconnected system of the form (3) is obtained with a particular structure. Figure 1 provides a graphical depiction of this reasoning. Then, the conditions in Theorem 1 can be used to establish ISS for the DDE (1). To this end, we define two types of Lyapunov functions. Let $\alpha_{1}, \alpha_{2} \in \mathscr{K}_{\infty}$ and let $v \in \mathscr{K}$.

\footnotetext{
${ }^{1}$ A function $\varphi: \mathbb{R}_{+} \rightarrow \mathbb{R}_{+}$is said to belong to class $\mathscr{G}$, i.e., $\varphi \in \mathscr{G}$, if it is continuous, non-decreasing and $\varphi(0)=0$, see [26].
} 


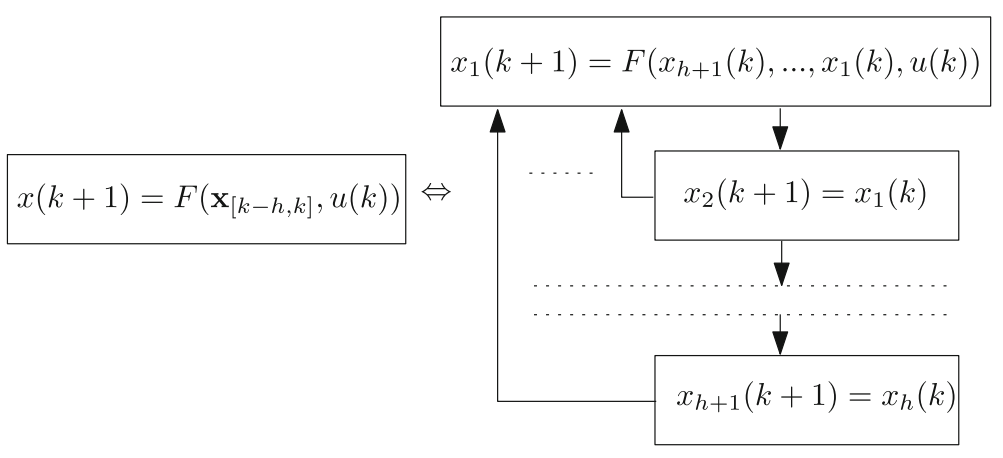

Fig. 1 A graphical depiction of the transformation of a DDE into an interconnected system

Definition 3 A function $V: \mathbb{R}^{n} \rightarrow \mathbb{R}_{+}$and some $\rho \in \mathscr{K}_{\infty}$ such that

$$
\begin{gathered}
\rho(s)<s, \quad \forall s \in \mathbb{R}_{>0}, \\
\alpha_{1}(\|x\|) \leq V(x) \leq \alpha_{2}(\|x\|), \quad \forall x \in \mathbb{R}^{n}, \\
V\left(F\left(\mathbf{x}_{[-h, 0]}, u\right)\right) \leq \max \left\{\max _{\left.\theta \in \mathbb{Z}_{[-h, 0]} \rho \circ V(x(\theta)), v(\|u\|)\right\},}\right.
\end{gathered}
$$

for all $\mathbf{x}_{[-h, 0]} \in\left(\mathbb{R}^{n}\right)^{h+1}$ and all $u \in \mathbb{R}^{m}$ is called an ISS-Lyapunov-Razumikhin function (ISS-LRF) for the DDE (1).

Furthermore, let $\bar{\alpha}_{1}, \bar{\alpha}_{2} \in \mathscr{K}_{\infty}$ and let $\bar{v} \in \mathscr{K}$.

Definition 4 A function $\bar{V}:\left(\mathbb{R}^{n}\right)^{h+1} \rightarrow \mathbb{R}_{+}$and some $\bar{\rho} \in \mathscr{K}_{\infty}$ such that $\bar{\rho}(s)<s$ for all $s \in \mathbb{R}_{>0}$ and that

$$
\begin{gathered}
\bar{\alpha}_{1}\left(\left\|\mathbf{x}_{[-h, 0]}\right\|\right) \leq \bar{V}\left(\mathbf{x}_{[-h, 0]}\right) \leq \bar{\alpha}_{2}\left(\left\|\mathbf{x}_{[-h, 0]}\right\|\right), \\
\bar{V}\left(\left\{\mathbf{x}_{[-h+1,0]}, F\left(\mathbf{x}_{[-h, 0]}, u\right)\right\}\right) \leq \max \left\{\bar{\rho} \circ \bar{V}\left(\mathbf{x}_{[-h, 0]}\right), \bar{v}(\|u\|)\right\},
\end{gathered}
$$

for all $\mathbf{x}_{[-h, 0]} \in\left(\mathbb{R}^{n}\right)^{h+1}$ and all $u \in \mathbb{R}^{m}$ is called an ISS-Lyapunov-Krasovskii function (ISS-LKF) for the DDE (1).

In [18, Theorem 3.5] it was established that the DDE (1) is ISS if it admits an ISS-LRF. The proof of this result relies on standard Lyapunov arguments, such as the ones used in [10], and the construction of a nonincreasing function that provides an upperbound on the ISS-LRF. Next, using Theorem 1, an alternative proof for the aforementioned result is provided. Moreover, as a by-product, an ISS-LKF is also obtained.

Theorem 2 Suppose that the DDE (1) admits an ISS-LRF and let V denote this ISSLRF. Then:

(i) the DDE (1) is ISS; 
(ii) there exist $\sigma_{i} \in \mathscr{K}_{\infty}, i \in \mathbb{Z}_{[1, h+1]}$, such that

$$
\bar{V}\left(\mathbf{x}_{[-h, 0]}\right):=\max _{\theta \in \mathbb{Z}_{[-h, 0]}} \sigma_{-\theta+1}^{-1} \circ V(x(\theta))
$$

is an ISS-LKF for the DDE (1).

Proof Let $x_{i}(k):=x(k-i+1)$ for all $i \in \mathbb{Z}_{[1, h+1]}$ and hence $N=h+1$. Then,

$$
\begin{aligned}
& g_{1}\left(x_{1}(k), \ldots, x_{h+1}(k), u(k)\right)=F\left(x_{h+1}(k), \ldots, x_{1}(k), u(k)\right), \\
& g_{i}\left(x_{1}(k), \ldots, x_{h+1}(k), u(k)\right)=x_{i-1}(k), \quad i \in \mathbb{Z}_{[2, h+1]} .
\end{aligned}
$$

Next, it is established that $V$ is an ISS-LF for all the subsystems (2), $i \in \mathbb{Z}_{[1, h+1]}$. Letting $W_{i}\left(x_{i}(k)\right)=V(x(k-i+1))$ for all $i \in \mathbb{Z}_{[1, h+1]}$, one obtains

$$
\gamma_{i, j}(s):= \begin{cases}\rho(s), & i=1, j \in \mathbb{Z}_{[1, h+1]} \\ \operatorname{Id}(s), & i \in \mathbb{Z}_{[2, h+1]}, j=i-1 \\ 0, & \text { otherwise. }\end{cases}
$$

Furthermore, $\alpha_{1, i}(s):=\alpha_{1}(s), \alpha_{2, i}(s):=\alpha_{2}(s), i \in \mathbb{Z}_{[1, h+1]}, \mu_{1}(s):=v(s)$ and $\mu_{i}(s):=0, i \in \mathbb{Z}_{[2, h+1]}$. As $\alpha_{1, i}, \alpha_{2, i} \in \mathscr{K}_{\infty}, \mu_{i} \in \mathscr{K} \cup\{0\}, \gamma_{i, j} \in \mathscr{K}_{\infty} \cup\{0\}$ for all $i, j \in \mathbb{Z}_{[1, h+1]}$ and as $\gamma_{i, i}(s)<s$ for all $s \in \mathbb{R}_{>0}$ (i.e., $\gamma_{1,1}(s)=\rho(s)$ and $\gamma_{i, i}(s)=0$ for all $i \neq 1$ ), it follows that, indeed, $V$ is an ISS-LF for all subsystems

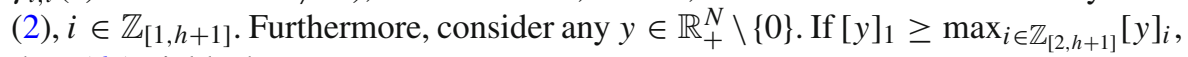
then (6a) yields that

$$
\max _{i \in \mathbb{Z}_{[1, h+1]}} \rho\left([y]_{i}\right)<[y]_{1} .
$$

Moreover, if $[y]_{1}<\max _{i \in \mathbb{Z}_{[2, h+1]}}[y]_{i}$, then there exists a $i(y) \in \mathbb{Z}_{[2, h+1]}$ such that

$$
[y]_{i-1}<[y]_{i} .
$$

As such, (4) holds and hence the hypothesis of Theorem 1 is satisfied. It then follows from claim (ii) of Theorem 1 that the DDE (1) is ISS, which establishes claim (i).

Furthermore, it follows from claim (i) of Theorem 1 that there exist $\sigma_{i} \in \mathscr{K}_{\infty}$, $i \in \mathbb{Z}_{[1, h+1]}$, such that the function $\bar{V}$ as defined in (8) is an ISS-LF for the interconnected system (3) with augmented state vector $x$ and hence the function (8) is an ISS-LKF for the DDE (1) with

$$
\begin{aligned}
& \bar{\alpha}_{1}(s):=\min _{i \in \mathbb{Z}_{[1, h+1]}} \sigma_{i}^{-1} \circ \alpha_{1}(s), \quad \bar{\alpha}_{2}(s):=\max _{i \in \mathbb{Z}_{[1, h+1]}} \sigma_{i}^{-1} \circ \alpha_{2}(s), \\
& \bar{\rho}(s):=\max _{i \in \mathbb{Z}_{[1, h+1]}} \max _{j \in \mathbb{Z}_{[1, h+1]}} \sigma_{i}^{-1} \circ \gamma_{i, j} \circ \sigma_{j}(s), \quad \bar{v}(s):=\sigma_{1}^{-1} \circ v(s) .
\end{aligned}
$$

Above, the construction of the functions $\bar{\rho}$ and $\bar{v}$ follows from the proof of Theorem 1 , which can be found in Appendix A. 
The proof of Theorem 2 shows that, for discrete-time systems, the Razumikhin method is an exact application of the small-gain theorem to DDEs. Indeed, (6a) guarantees fulfillment of the small-gain condition (4). The above observation and the approach used to prove Theorem 2 are a discrete-time counterpart to the results in [27], where a Razumikhin theorem for continuous-time systems was proven using small-gain arguments.

Remark 3 If a DDE is ISS, it is also globally asymptotically stable (GAS), i.e., for $u(k)=0$ for all $k \in \mathbb{Z}_{+}$. Therefore, Theorem 2 also provides a different proof for [19, Theorem 3.2] and its extension to delay difference inclusions, i.e., [4, Theorem 3.8]. Moreover, the results therein are generalized in the sense that $\rho$ is a nonlinear function rather than a linear function. Further results in this paper have similar implications when GAS rather than ISS is of concern.

As it was also indicated above, an explicit expression for the functions $\sigma_{i} \in \mathscr{K}_{\infty}$, $i \in \mathbb{Z}_{[1, h+1]}$, in Theorem 1 can be obtained using the ideas presented in [12]. In what follows, based on a suitable construction of the functions $\sigma_{i}$ an explicit expression for an ISS-LKF constructed from an ISS-LRF is presented.

Theorem 3 Suppose that the DDE (1) admits an ISS-LRF, i.e., V. Then, an ISS-LKF for (1) is given by

$$
\bar{V}\left(\mathbf{x}_{[-h, 0]}\right):=\max _{\theta \in \mathbb{Z}_{[-h, 0]}} \rho_{h+1+\theta} \circ V(x(\theta))
$$

where $\rho_{i}(s)=\frac{\rho(s)+i s}{i+1}, i \in \mathbb{Z}_{[1, h]}$, and $\rho_{h+1}(s)=s$.

Proof Note that, by definition, $\rho_{i} \in \mathscr{K}_{\infty}$ for all $i \in \mathbb{Z}_{[1, h+1]}$. Next, it is established that

$$
\rho(s)<\rho_{1}(s)<\cdots<\rho_{h}(s)<\rho_{h+1}(s)=s .
$$

As $\rho(s)<s$ it holds that

$$
\rho(s)<s=(i+1)^{2} s-(i+2) i s,
$$

for all $s \in \mathbb{R}_{>0}$. The above is equivalent to

$$
(i+2)(\rho(s)+i s)<(i+1)(\rho(s)+(i+1) s)
$$

which implies that $\rho_{i}(s)<\rho_{i+1}(s)$, for all $i \in \mathbb{Z}_{[1, h]}$ and all $s \in \mathbb{R}_{>0}$. Obviously, $\rho_{i}(s)<\frac{s+i s}{i+1}=s$, which establishes that (10) holds. Next, let $\pi_{i}(s):=\rho_{i-1} \circ \rho_{i}^{-1}(s)$, $i \in \mathbb{Z}_{[1, h+1]}$ and $s \in \mathbb{R}_{+}$, with $\rho_{0}(s):=\rho(s)$. Then, as $\rho_{i-1}(s)<\rho_{i}(s)$ it follows that $\pi_{i}(s)=\rho_{i-1} \circ \rho_{i}^{-1}(s)<\rho_{i} \circ \rho_{i}^{-1}(s)=s$. Letting $\pi(s):=\max _{i \in \mathbb{Z}_{[1, h+1]}} \pi_{i}(s)$, yields that $\pi(s)<s$ for all $s \in \mathbb{R}_{>0}$. 
Consider any $\mathbf{x}_{[-h, 0]} \in\left(\mathbb{R}^{n}\right)^{h+1}$ and any $u \in \mathbb{R}^{m}$. Then,

$$
\begin{aligned}
& \bar{V}\left(\left\{\mathbf{x}_{[-h+1,0]}, F\left(\mathbf{x}_{[-h, 0]}, u\right)\right\}\right) \\
& =\max \left\{\rho_{h+1} \circ V\left(F\left(\mathbf{x}_{[-h, 0]}, u\right)\right), \max _{\theta \in \mathbb{Z}_{[-h+1,0]}} \rho_{h+\theta} \circ V(x(\theta))\right\} \\
& \leq \max \left\{\max _{\theta \in \mathbb{Z}_{[-h, 0]}} \rho \circ V(x(\theta)), \max _{\theta \in \mathbb{Z}_{[-h+1,0]}} \rho_{h+\theta} \circ V(x(\theta)), v(\|u\|)\right\} \\
& =\max \left\{\rho \circ V(x(-h)), \max _{\theta \in \mathbb{Z}_{[-h+1,0]}} \rho_{h+\theta} \circ V(x(\theta)), v(\|u\|)\right\} \\
& =\max \left\{\max _{\theta \in \mathbb{Z}_{[-h, 0]}} \pi_{h+\theta+1} \circ \rho_{h+\theta+1} \circ V(x(\theta)), v(\|u\|)\right\} \\
& \leq \max \left\{\max _{\theta \in \mathbb{Z}_{[-h, 0]}} \pi \circ \rho_{h+\theta+1} \circ V(x(\theta)), v(\|u\|)\right\} \\
& =\max \left\{\pi \circ \bar{V}\left(\mathbf{x}_{[-h, 0]}\right), v(\|u\|)\right\} .
\end{aligned}
$$

Let $\bar{\rho}(s):=\pi(s), \bar{\alpha}_{1}(s):=\min _{i \in \mathbb{Z}_{[1, h+1]}} \rho_{i} \circ \alpha_{1}(s), \bar{\alpha}_{2}(s):=\max _{i \in \mathbb{Z}_{[1, h+1]}} \rho_{i} \circ \alpha_{2}(s)$ and $\bar{v}(s):=v(s)$. As $\bar{\alpha}_{1}, \bar{\alpha}_{2} \in \mathscr{K}_{\infty}, \bar{v} \in \mathscr{K}, \bar{\rho} \in \mathscr{K}_{\infty}$ and $\bar{\rho}(s)<s$ for all $s \in \mathbb{R}_{>0}$, it follows that $\bar{V}$ is an ISS-LKF for the DDE (1).

Starting from the ISS-LKF (9), it can be established, using standard Lyapunov arguments, see, e.g., [10], that the DDE (1) is ISS.

Remark 4 Theorem 4.1 in [4] presents, under the assumption that $\rho(s)=\kappa s$ for some $\kappa \in \mathbb{R}_{[0,1)}$, a method to construct a LKF from a LRF. Theorem 3 recovers the aforementioned result as a particular case.

Theorem 3 establishes that, for discrete-time systems, any ISS-LRF can be used to construct an ISS-LKF. This is in accordance with the results for continuous-time systems in, e.g., [7,13], which show that the conditions for stability provided by the Razumikhin method are stronger than those provided by the Krasovskii approach. However, the Razumikhin method, on the other hand, requires the construction of a function on $\mathbb{R}^{n}$ rather than a function on $\left(\mathbb{R}^{n}\right)^{h+1}$, as is the case for the Krasovskii approach. Therefore, it can be concluded that the Razumikhin method is computationally more attractive but conceptually more conservative than the Krasovskii approach. The interested reader is referred to $[4,13]$ for a more detailed discussion of the Razumikhin method versus the Krasovskii approach. Furthermore, the techniques presented in [3] and the references therein can be used to obtain local variants of the results presented in this section, which can be used to handle DDEs that are subject to constraints.

\section{ISS analysis for interconnected systems with interconnection delays}

In the previous section, it was established that a DDE can be transformed into an interconnection of difference equations without delay. Then, the small-gain theorem can 
be used for the ISS analysis of the so-obtained interconnected system. In the present section, this technique will be applied to establish ISS for interconnected systems with interconnection delays. Interconnected systems with local delays will be treated in the next section.

Consider the subsystems (2) but now with interconnection delays, i.e., for all $i \in$ $\mathbb{Z}_{[1, N]}$,

$$
x_{i}(k+1)=g_{i}\left(x_{1}\left(k-h_{i, 1}\right), \ldots, x_{N}\left(k-h_{i, N}\right), u(k)\right), \quad k \in \mathbb{Z}_{+},
$$

with $x_{i}(k) \in \mathbb{R}^{n_{i}}, u(k) \in \mathbb{R}^{m}$ and $g_{i}$ as defined in (2). Moreover, $h_{i, j} \in \mathbb{Z}_{+}, i, j \in$ $\mathbb{Z}_{[1, N]}$, is the interconnection delay from subsystem $j$ to subsystem $i$. In this section, it is assumed that $h_{i, i}=0$ for all $i \in \mathbb{Z}_{[1, N]}$, i.e., the subsystems are not affected by local delays. To describe the complete interconnected system with interconnection delays, let $x:=\operatorname{col}\left(\left\{x_{l}\right\}_{\left.l \in \mathbb{Z}_{[1, N]}\right)} \in \mathbb{R}^{n}\right.$, which yields a system of the form (1) where

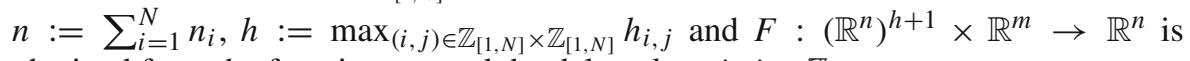
obtained from the functions $g_{i}$ and the delays $h_{i, j}, i, j \in \mathbb{Z}_{[1, N]}$.

The ISS analysis for the interconnected system (1) obtained from (11) using traditional techniques for delay systems is hampered by the size and complexity of the overall system. In what follows, it is established that the ISS analysis can be greatly simplified using Theorem 1.

Theorem 4 Suppose that the subsystems (2) satisfy the hypothesis of Theorem 1. Then, the interconnected system (1) obtained from the subsystems with interconnection delays (11) is ISS.

Proof The proof consists of three parts. In the first part, the interconnected system with interconnection delays is transformed into an augmented interconnected system without delay. Therefore, consider the following procedure. Let $I=1, J=2$ and let $\bar{h}:=\sum_{i=1}^{N} \sum_{j=1}^{N} h_{i, j}$. If $h_{I, J} \geq 1$, let

$$
\hat{g}_{N+1}\left(x_{1}, \ldots, x_{N+\bar{h}}, u\right):=x_{J}, \quad \text { and } \quad W_{N+1}\left(x_{N+1}\right):=W_{J}\left(x_{N+1}\right) .
$$

Note that, the above definition corresponds to the case where $x_{N+1}(k):=x_{J}(k-1)$ for all $k \in \mathbb{Z}_{+}$. Furthermore, if $h_{I, J} \geq 2$, let

$$
\hat{g}_{N+l+1}\left(x_{1}, \ldots, x_{N+\bar{h}}, u\right):=x_{N+l}, \quad \text { and } \quad W_{N+l+1}\left(x_{N+l+1}\right):=W_{J}\left(x_{N+l+1}\right)
$$

for all $l \in \mathbb{Z}_{\left[1, h_{I, J}-1\right]}$. Repeat this procedure for all $I, J \in \mathbb{Z}_{[1, N]}$ and $I \neq J$ such that the interconnection delays in (11) are replaced by new subsystems. Thus, an interconnection of $N+\bar{h}$ subsystems without delay is obtained, i.e.,

$$
x_{i}(k+1)=\hat{g}_{i}\left(x_{1}(k), \ldots, x_{N+\bar{h}}(k), u(k)\right), \quad k \in \mathbb{Z}_{+},
$$

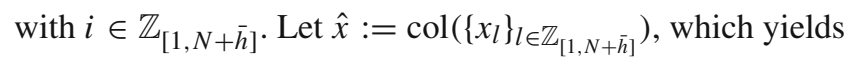

$$
\hat{x}(k+1)=\hat{G}(\hat{x}(k), u(k)), \quad k \in \mathbb{Z}_{+} .
$$




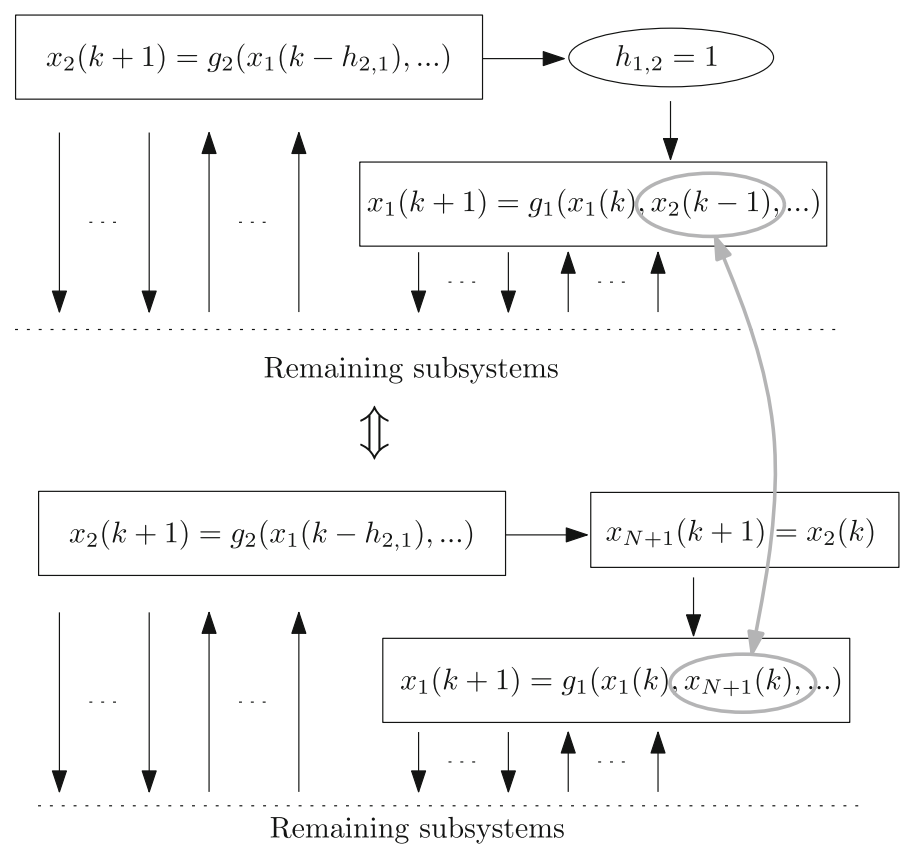

Fig. 2 A graphical depiction of the transformation of an interconnected system with interconnection delays into an augmented interconnected system without delay

The transformation of the interconnected system with interconnection delays into the augmented interconnected system without delay is shown in Fig. 2.

In the second part of the proof it is shown that $W_{i}$ is an ISS-LF for subsystem (12) for all $i \in \mathbb{Z}_{[1, N+\bar{h}]}$. Moreover, it is also shown that, if the interconnected system (1) obtained from (11) satisfies the small-gain condition (4), then the augmented interconnected system (13) satisfies a similar small-gain condition. It follows from the hypotheses of Theorem 1 that $W_{i}$ is an ISS-LF for subsystem (12), for all $i \in \mathbb{Z}_{[1, N]}$. Furthermore, if $h_{1,2} \geq 1$, then it follows, by definition, that

$$
W_{N+1}\left(\hat{g}_{N+1}\left(x_{1}, \ldots, x_{N+\bar{h}}, u\right)\right)=W_{2}\left(x_{2}\right) .
$$

Hence, let $W_{N+1}\left(x_{2}\right):=W_{2}\left(x_{2}\right)$ for all $x_{2} \in \mathbb{R}^{n_{2}}$. If $h_{1,2} \geq 2$, then it follows, again by definition, that

$$
W_{N+l+1}\left(\hat{g}_{N+l+1}\left(x_{1}, \ldots, x_{N+\bar{h}}, u\right)\right)=W_{N+l}\left(x_{N+l}\right),
$$

for all $l \in \mathbb{Z}_{\left[1, h_{I, J}-1\right]}$. Hence, let $W_{N+l+1}\left(x_{2}\right):=W_{2}\left(x_{2}\right)$ for all $x_{2} \in \mathbb{R}^{n_{2}}$. Similarly, it can be shown that $W_{i}$ is an ISS-LF for subsystem (12) for all $i \in \mathbb{Z}_{[N+1, N+\bar{h}]}$. Next, the corresponding gain functions $\gamma_{i, j}$ are defined recursively and it is shown that they satisfy a small-gain condition. Therefore, let $\gamma_{i, j}^{0}:=\gamma_{i, j}$ for all $i, j \in \mathbb{Z}_{[1, N]}$. Furthermore, let $(I, J) \in \mathbb{Z}_{[1, N+l]} \times \mathbb{Z}_{[1, N+l]}$ correspond to the interconnection with delay between subsystem $J$ and $I$ for which the new state $x_{N+l+1}$ was introduced. Define 


$$
\gamma_{i, j}^{l+1}:= \begin{cases}0, & i=I, j=J \text { or } i \neq I, j=N+l+1 \\ & \text { or } i=N+l+1, j \neq J \\ \gamma_{I, J}^{l}, & i=I, j=N+l+1 \\ \mathrm{Id}, & i=N+l+1, j=J \\ \gamma_{i, j}^{l}, & \text { otherwise, }\end{cases}
$$

for all $i, j \in \mathbb{Z}_{[1, N+l+1]}$ and all $l \in \mathbb{Z}_{[0, \bar{h}-1]}$. In what follows, it is proven, by induction, that for all $l \in \mathbb{Z}_{[0, \bar{h}]}$ and all $y \in \mathbb{R}_{+}^{N+l} \backslash\{0\}$, there exists a $i(y) \in \mathbb{Z}_{[1, N+l]}$ such that

$$
\max _{j \in \mathbb{Z}_{[1, N+l]}} \gamma_{i, j}^{l}\left([y]_{j}\right)<[y]_{i} .
$$

Therefore, let $l=0$ and let $y:=\left[\bar{y}^{\top} \tilde{y}\right]^{\top}$ for any $\bar{y} \in \mathbb{R}_{+}^{N}$ and $\tilde{y} \in \mathbb{R}_{+}$such that $y \neq 0$. If $\tilde{y} \leq[\bar{y}]_{J}$, then it follows from (4) that (14) with $l=1$ holds for $i(y)=i(\bar{y})$. Conversely, if $\tilde{y}>[\bar{y}]_{J}$, then

$$
\max _{j \in \mathbb{Z}_{[1, N+1]}} \gamma_{N+1, j}^{1}\left([y]_{j}\right)=[\bar{y}]_{J}<\tilde{y}=[y]_{N+1} .
$$

Thus, it has been established that (14) with $l=1$ holds for $i(y)=N+1$. Next, consider any $\ell \in \mathbb{Z}_{[0, \bar{h}-1]}$ and suppose that (14) with $l=\ell$ holds, i.e., for all $\bar{y} \in \mathbb{R}_{+}^{N+\ell}$ there exists some $i(\bar{y})$ such that (14) holds. Let $y:=\left[\begin{array}{ll}\bar{y}^{\top} & \tilde{y}\end{array}\right]^{\top}$ for any $\bar{y} \in \mathbb{R}_{+}^{N+\ell}$ and $\tilde{y} \in \mathbb{R}_{+}$such that $y \neq 0$. If $\tilde{y} \leq[\bar{y}]_{J}$, then it follows from (14) with $l=\ell$ that (14) with $l=\ell+1$ also holds for $i(y)=i(\bar{y})$. Conversely, if $\tilde{y}>[\bar{y}]_{J}$, then

$$
\max _{j \in \mathbb{Z}_{[1, N+\ell+1]}} \gamma_{N+\ell+1, j}^{\ell+1}\left([y]_{j}\right)=[\bar{y}]_{J}<\tilde{y}=[y]_{N+\ell+1} .
$$

Hence, (14) with $l=\ell+1$ holds for $i(y)=N+\ell$. Thus, it has been established, by induction, that (14) holds for any $l \in \mathbb{Z}_{[0, \bar{h}]}$. Therefore, the ISS-LFs $W_{i}$ corresponding to the subsystems (12) satisfy the small-gain condition (4) and it follows from Theorem 1 that the augmented interconnected system (13) is ISS.

In the third part of the proof it is shown that the interconnected system with interconnection delays, i.e., (1) obtained from (11), is ISS if the augmented interconnected system (13) is ISS. The proof of this claim is similar to the proof of Lemma 3.3 in [4] and is therefore omitted for brevity.

Theorem 4 establishes that, if the delay-independent small-gain condition (4) holds, finite interconnection delays cannot cause input-to-state instability of an interconnected system. Hence, the ISS analysis of interconnected systems with interconnection delays can be reduced, via Theorem 4, to the ISS analysis of standard interconnected systems. The above discussion indicates an advantage of considering interconnection delays and local delays separately as opposed to considering both types of delay at once, as done in other works, see, e.g., [1,28]. Note that, when interconnection delays are not present, the results in this paper parallel the continuous-time results in the aforementioned references. 
Remark 5 For continuous-time systems a similar relation was also observed in [23, $25,29]$ and in the context of integral ISS in $[8,9]$. However, the derivations for continuous-time systems rely on different arguments. Indeed, the transformation applied to prove Theorem 4 leads to an infinite dimensional system for the continuous-time case.

Remark 6 Theorem 4 does not assume any knowledge about the interconnection delay. If the interconnection delay is assumed to be known, potentially less conservative delay-dependent small-gain conditions can be derived, see, e.g., [8] for the continuous-time case.

\section{ISS analysis for interconnected systems with local delays}

Another cause for delay in interconnected systems are inherent delays in the dynamical process in one or more of the subsystems. Therefore, in this section, the techniques developed in Theorems 1 and 2 will be used to establish ISS for interconnected systems with local delays.

Consider an interconnection of $N \in \mathbb{Z}_{\geq 2}$ subsystems affected by local delays. The dynamics of the $i$ th subsystem, $i \in \mathbb{Z}_{[1, N]}$, is described by

$$
x_{i}(k+1)=f_{i}\left(\mathbf{x}_{[k-\hat{h}, k] ; i}, x_{1}(k), \ldots, x_{N}(k), u(k)\right), \quad k \in \mathbb{Z}_{+},
$$

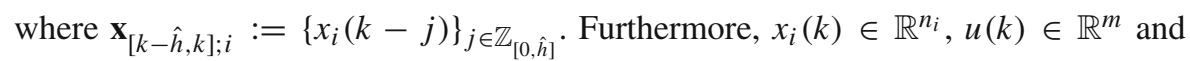
$f_{i}:\left(\mathbb{R}^{n_{i}}\right)^{\hat{h}+1} \times \mathbb{R}^{n_{1}} \times \cdots \times \mathbb{R}^{n_{N}} \times \mathbb{R}^{m} \rightarrow \mathbb{R}^{n_{i}}, i \in \mathbb{Z}_{[1, N]}$. Above, $\hat{h} \in \mathbb{Z}_{+}$is the maximal delay affecting (15). Note that, with a slight abuse of notation, to simplify the exposition, $x_{i}(k)$ appears twice as an argument of $f_{i}$. Moreover, it can be assumed without any restriction to the generality of the results that all subsystems (15) share the same maximal delay. To describe the complete interconnected system obtained from the subsystems with local delays (15), let $x:=\operatorname{col}\left(\left\{x_{l}\right\}_{l \in \mathbb{Z}_{[1, N]}}\right) \in \mathbb{R}^{n}$, which yields an interconnected system of the form (1) where $h:=\hat{h}, n:=\sum_{i=1}^{N} n_{i}$ and $F$ is obtained from $f_{i}, i \in \mathbb{Z}_{[1, N]}$, i.e., $F\left(\mathbf{x}_{[-h, 0]}, u\right)=\operatorname{col}\left(\left\{f_{i}\left(\mathbf{x}_{[-h, 0] ; i}, x_{1}(0), \ldots, x_{N}(0), u\right)\right\}_{i \in \mathbb{Z}_{[1, N]}}\right)$.

Next, let $\alpha_{1, i}, \alpha_{2, i} \in \mathscr{K}_{\infty}, \mu_{i} \in \mathscr{K} \cup\{0\}$, for all $i \in \mathbb{Z}_{[1, N]}$, and let $\gamma_{i, j} \in \mathscr{K}_{\infty} \cup\{0\}$ for all $i, j \in \mathbb{Z}_{[1, N]}$. Then, consider the functions $W_{j}: \mathbb{R}^{n_{j}} \rightarrow \mathbb{R}_{+}, j \in \mathbb{Z}_{[1, N]}$, that satisfy

$$
\alpha_{1, j}\left(\left\|x_{j}\right\|\right) \leq W_{j}\left(x_{j}\right) \leq \alpha_{2, j}\left(\left\|x_{j}\right\|\right), \quad \forall x_{j} \in \mathbb{R}^{n_{j}}
$$

Definition 5 Let $\gamma_{i, i}(s)<s$ for all $s \in \mathbb{R}_{>0}$. A function $W_{i}, i \in \mathbb{Z}_{[1, N]}$, that satisfies

$$
\begin{aligned}
& W_{i}\left(f_{i}\left(\mathbf{x}_{[-h, 0] ; i}, x_{1}, \ldots, x_{N}, u\right)\right) \\
& \quad \leq \max \left\{\max _{\theta \in \mathbb{Z}_{[-h, 0]}} \gamma_{i, i} \circ W_{i}\left(x_{i}(\theta)\right), \max _{j \in \mathbb{Z}_{[1, N]}, j \neq i} \gamma_{i, j} \circ W_{j}\left(x_{j}\right), \mu_{i}(\|u\|)\right\},
\end{aligned}
$$

for all $\mathbf{x}_{[-h, 0] ; i} \in\left(\mathbb{R}^{n_{i}}\right)^{h+1}, x_{j} \in \mathbb{R}^{n_{j}}, j \in \mathbb{Z}_{[1, N]}$ and $j \neq i, x_{i}:=x_{i}(0)$ and all $u \in \mathbb{R}^{m}$ is called an ISS-Lyapunov-Razumikhin function (ISS-LRF) for subsystem (15). 
Note that Definition 5 is a generalization of Definition 3 for interconnected systems with local delays.

Theorem 5 Suppose that all subsystems (15), $i \in \mathbb{Z}_{[1, N]}$, admit an ISS-LRF. Furthermore, suppose that for all $y \in \mathbb{R}_{+}^{N} \backslash\{0\}$, there exists a $i(y) \in \mathbb{Z}_{[1, N]}$ such that

$$
\max _{j \in \mathbb{Z}_{[1, N]}} \gamma_{i, j}\left([y]_{j}\right)<[y]_{i}
$$

Then, the following claims hold:

(i) There exist $\sigma_{i} \in \mathscr{K}_{\infty}, i \in \mathbb{Z}_{[1, N]}$, such that

$$
W(x)=\max _{i \in \mathbb{Z}} \sigma_{i 1, N]}^{-1} \circ W_{i}\left(x_{i}\right)
$$

is an ISS-LRF for the interconnected system (1) obtained from (15);

(ii) The interconnected system (1) obtained from (15) is ISS.

Proof The proof of Theorem 5 is similar to the proof of Theorem 1. It follows from the small-gain condition (16) that the functions $\gamma_{i, j}$ satisfy the hypothesis of Lemma 1. Given $\sigma_{i} \in \mathscr{K}_{\infty}, i \in \mathbb{Z}_{[1, N]}$, let $\mu(s):=\max _{i \in \mathbb{Z}_{[1, N]}} \sigma_{i}^{-1} \circ \mu_{i}(s)$ for all $s \in \mathbb{R}_{+}$and let

$$
\gamma(s):=\max _{i \in \mathbb{Z}_{[1, N]}} \max _{j \in \mathbb{Z}_{[1, N]}} \sigma_{i}^{-1} \circ \gamma_{i, j} \circ \sigma_{j}(s)
$$

Then, it follows from Fact 1 in Appendix A that $\gamma \in \mathscr{K}_{\infty}$ and $\mu \in \mathscr{K}$. Moreover, Lemma 1 yields that $\gamma(s)<s$ for all $s \in \mathbb{R}_{>0}$.

Next, consider the candidate ISS-LRF (17). Then

$$
\begin{aligned}
& W\left(F\left(\mathbf{x}_{[-h, 0]}, u\right)\right)=\max _{i \in \mathbb{Z}_{[1, N]}} \sigma_{i}^{-1} \circ W_{i}\left(f_{i}\left(\mathbf{x}_{[-h, 0] ; i}, x_{1}(0), \ldots, x_{N}(0), u\right)\right) \\
& \leq \max _{i \in \mathbb{Z}_{[1, N]}} \sigma_{i}^{-1} \circ \max \left\{\max _{\theta \in \mathbb{Z}_{[-h, 0]}} \gamma_{i, i} \circ W_{i}\left(x_{i}(\theta)\right), \max _{j \in \mathbb{Z}_{[1, N]}, j \neq i} \gamma_{i, j} \circ W_{j}\left(x_{j}(0)\right), \mu_{i}(\|u\|)\right\} \\
& \leq \max \left\{\max _{\theta \in \mathbb{Z}_{[-h, 0]}} \max _{i \in \mathbb{Z}_{[1, N]}} \max _{j \in \mathbb{Z}_{[1, N]}} \sigma_{i}^{-1} \circ \gamma_{i, j} \circ \sigma_{j} \circ \sigma_{j}^{-1} \circ W_{j}\left(x_{j}(\theta)\right), \mu(\|u\|)\right\} \\
& \leq \max \left\{\max _{\theta \in \mathbb{Z}_{[-h, 0]}} \gamma \circ \max _{i^{\prime} \in \mathbb{Z}_{[1, N]}} \sigma_{i^{\prime}}^{-1} \circ W_{i^{\prime}}\left(x_{i^{\prime}}(\theta)\right), \mu(\|u\|)\right\} \\
& =\max \left\{\max _{\theta \in \mathbb{Z}_{[-h, 0]}} \gamma \circ W(x(\theta)), \mu(\|u\|)\right\} \text {, }
\end{aligned}
$$

for all $\left(\mathbf{x}_{[-h, 0]}, u\right) \in\left(\mathbb{R}^{n}\right)^{h+1} \times \mathbb{R}^{m}$. Furthermore, the equivalence of norms [14] yields that there exist some $c_{1}, c_{2} \in \mathbb{R}_{>0}$ such that $c_{1} \max _{i \in \mathbb{Z}_{[1, N]}}\left\|x_{i}\right\| \leq\|x\| \leq$ $c_{2} \max _{i \in \mathbb{Z}_{[1, N]}}\left\|x_{i}\right\|$. Hence, the $\mathscr{K}_{\infty}$ bounds for the functions $W_{i}, i \in \mathbb{Z}_{[1, N]}$, yield

$$
\min _{i \in \mathbb{Z}_{[1, N]}} \sigma_{i}^{-1} \circ \alpha_{1, i}\left(c_{2}^{-1}\|x\|\right) \leq W(x) \leq \max _{i \in \mathbb{Z}_{[1, N]}} \sigma_{i}^{-1} \circ \alpha_{2, i}\left(c_{1}^{-1}\|x\|\right) .
$$


Therefore, let $\alpha_{1}(s):=\min _{i \in \mathbb{Z}_{[1, N]}} \sigma_{i}^{-1} \circ \alpha_{1, i}\left(c_{2}^{-1} s\right)$ and let $\alpha_{2}(s):=\max _{i \in \mathbb{Z}_{[1, N]}} \sigma_{i}^{-1}$ 。 $\alpha_{2, i}\left(c_{1}^{-1} s\right)$. As it follows from Fact 1 that $\alpha_{1}, \alpha_{2} \in \mathscr{K}_{\infty}, W$ is an ISS-LRF for the interconnected system (1) obtained from (15), which establishes claim (i). Observing that claim (ii) follows directly from claim (i) and Theorem 2 completes the proof.

Thus, it has been established that if all subsystems with local delays (15) admit an ISS-LRF and the small-gain condition (16) holds, then the interconnected system (1) obtained from (15) admits an ISS-LRF. Moreover, as a direct consequence, it also follows that the interconnected system is ISS.

The following result, which is based on the Krasovskii approach, is similar to Theorem 5. Let $\bar{\alpha}_{1, i}, \bar{\alpha}_{2, i} \in \mathscr{K}_{\infty}, \bar{\mu}_{i} \in \mathscr{K} \cup\{0\}$, for all $i \in \mathbb{Z}_{[1, N]}$, and let $\bar{\gamma}_{i, j} \in \mathscr{K}_{\infty} \cup\{0\}$ for all $i, j \in \mathbb{Z}_{[1, N]}$. Then, consider the functions $\bar{W}_{j}:\left(\mathbb{R}^{n_{j}}\right)^{(h+1)} \rightarrow \mathbb{R}_{+}, j \in \mathbb{Z}_{[1, N]}$, that satisfy

$$
\bar{\alpha}_{1, j}\left(\left\|\mathbf{x}_{[-h, 0] ; j}\right\|\right) \leq \bar{W}_{j}\left(\mathbf{x}_{[-h, 0] ; j}\right) \leq \bar{\alpha}_{2, j}\left(\left\|\mathbf{x}_{[-h, 0] ; j}\right\|\right), \quad \forall \mathbf{x}_{[-h, 0] ; j} \in\left(\mathbb{R}^{n_{j}}\right)^{h+1} .
$$

Definition 6 Let $\bar{\gamma}_{i, i}(s)<s$ for all $s \in \mathbb{R}_{>0}$. A function $\bar{W}_{i}, i \in \mathbb{Z}_{[1, N]}$, that satisfies

$$
\begin{aligned}
& \bar{W}_{i}\left(\left\{\mathbf{x}_{[-h+1,0] ; i}, f_{i}\left(\mathbf{x}_{[-h, 0] ; i}, x_{1}(0), \ldots, x_{N}(0), u\right)\right\}\right) \\
& \quad \leq \max \left\{\max _{j \in \mathbb{Z}_{[1, N]}} \bar{\gamma}_{i, j} \circ \bar{W}_{j}\left(\mathbf{x}_{[-h, 0] ; j}\right), \bar{\mu}_{i}(\|u\|)\right\},
\end{aligned}
$$

for all $\mathbf{x}_{[-h, 0] ; j} \in\left(\mathbb{R}^{n_{j}}\right)^{h+1}, j \in \mathbb{Z}_{[1, N]}$, and all $u \in \mathbb{R}^{m}$ is called an ISS-LyapunovKrasovskii function (ISS-LKF) for subsystem (2).

As it was also the case for the Razumikhin method, Definition 6 is a generalization of Definition 4 for interconnected systems with local delays.

Theorem 6 Suppose that all subsystems (15), $i \in \mathbb{Z}_{[1, N]}$, admit an ISS-LKF. Furthermore, suppose that for all $y \in \mathbb{R}_{+}^{N} \backslash\{0\}$, there exists a $i(y) \in \mathbb{Z}_{[1, N]}$ such that

$$
\max _{j \in \mathbb{Z}_{[1, N]}} \bar{\gamma}_{i, j}\left([y]_{j}\right)<[y]_{i} .
$$

Then, the following claims hold:

(i) There exist $\bar{\sigma}_{i} \in \mathscr{K}_{\infty}, i \in \mathbb{Z}_{[1, N]}$, such that

$$
\bar{W}\left(\mathbf{x}_{[-h, 0]}\right)=\max _{i \in \mathbb{Z}_{[1, N]}} \bar{\sigma}_{i}^{-1} \circ \bar{W}_{i}\left(\mathbf{x}_{[-h, 0] ; i}\right)
$$

is an ISS-LKF for the interconnected system (1) obtained from (15);

(ii) The interconnected system (1) obtained from (15) is ISS.

Proof Using the definitions

$$
\begin{aligned}
\bar{\alpha}_{1}(s) & :=\min _{i \in \mathbb{Z}_{[1, N]}} \bar{\sigma}_{i}^{-1} \circ \bar{\alpha}_{1, i}\left(c_{2}^{-1} s\right), \quad \bar{\alpha}_{2}(s):=\max _{i \in \mathbb{Z}_{[1, N]}} \bar{\sigma}_{i}^{-1} \circ \bar{\alpha}_{2, i}\left(c_{1}^{-1} s\right), \\
\bar{\gamma}(s) & :=\max _{i \in \mathbb{Z}_{[1, N]}} \max _{j \in \mathbb{Z}_{[1, N]}} \bar{\sigma}_{i}^{-1} \circ \bar{\gamma}_{i, j} \circ \bar{\sigma}_{j}(s), \quad \bar{\mu}(s):=\max _{i \in \mathbb{Z}_{[1, N]}} \bar{\sigma}_{i}^{-1} \circ \bar{\mu}_{i}(s),
\end{aligned}
$$


for some $c_{1}, c_{2} \in \mathbb{R}_{>0}$, the proof of Theorem 6 can be obtained using a reasoning similar to the reasoning used in the proofs of Theorem 1 and Theorem 5.

The advantages of standard Krasovskii and Razumikhin theorems when compared to each other, see the discussion at the end of Sect. 3, also apply to Theorem 5 and Theorem 6, i.e., computational simplicity is traded for conceptual generality. In fact, three options can be distinguished for interconnected systems with local delay, i.e., (i) consider the interconnected system as a single system with delay and apply the Krasovskii approach; (ii) consider the interconnected system as an interconnection of several subsystems with delay, apply the Krasovskii approach locally and then use small-gain arguments (see Theorem 6); (iii) consider, based on the transformation developed in Sect. 3, the interconnected system with delay as the interconnection of a very large set of subsystems without delay and apply small-gain arguments directly (see Theorem 5 and the observations in Sect. 3). Due to the large size and complexity of interconnected systems (i) is generally not a tractable approach. Both approaches (ii) and (iii) lead to a tractable ISS analysis framework for large-scale systems. Furthermore, while the conditions related to (iii) are more conservative, these conditions are simpler to verify compared to the conditions related to (ii).

Remark 7 Theorems 5 and 6 are discrete-time counterparts of Theorem 3.4 and Theorem 3.7 in [1], respectively. However, the reasoning required to prove the results for the discrete-time case differs significantly with respect to the continuous-time case, mainly due to the different conditions involved in the Razumikhin method. As such, Theorems 5 and 6 provide a valuable addition to the results presented in [1].

Remark 8 Suppose that, for the subsystems with local delay (15), for all $i \in \mathscr{I} \subset$ $\mathbb{Z}_{[1, N]}, W_{i}$ is an ISS-LRF. Furthermore, suppose for all $i \in \mathbb{Z}_{[1, N]} \backslash \mathscr{I}, \bar{W}_{i}$ is an ISS-LKF. Then, an ISS-LKF can be constructed for the subsystems (15) for all $i \in \mathscr{I}$ via Theorem 3. Thus, the ISS analysis for the interconnected system (1) obtained from (15) can be performed via Theorem 6.

In Theorem 4 small-gain arguments are used to establish robustness of ISS with respect to interconnection delays for an interconnected system with disturbance inputs. On the other hand, in Theorems 5 and 6 small-gain arguments are used to establish robustness of ISS with respect to inputs from the other subsystems for a subsystem with local delays and disturbance inputs. Hence, while all proofs are based on small-gain arguments, the reasoning used to prove Theorem 4 is different from the reasoning used to prove Theorems 5 and 6 . The fact that the reasoning of Theorem 4 also applies in the context of Theorems 5 and 6 is exploited in the next section.

\section{ISS analysis for interconnected systems with delay}

If an interconnected system contains one or more subsystems with delays in the dynamical process and the subsystems are located in different geographical places, a general interconnected system with delay is obtained. For the ISS analysis of such systems, a combination of Theorem 4 with Theorems 5 or 6 , respectively, is required. In this section, such results are derived. 
Consider an interconnection of $N \in \mathbb{Z}_{\geq 2}$ subsystems with local delays described by (15) subject to interconnection delays. Then, the dynamics of the $i$ th subsystem, $i \in \mathbb{Z}_{[1, N]}$, is given by

$$
x_{i}(k+1)=f_{i}\left(\mathbf{x}_{[k-\hat{h}, k] ; i}, x_{1}\left(k-h_{i, 1}\right), \ldots, x_{N}\left(k-h_{i, N}\right), u(k)\right), \quad k \in \mathbb{Z}_{+},
$$

with $x_{i}(k) \in \mathbb{R}^{n_{i}}, u(k) \in \mathbb{R}^{m}$ and $f_{i}, i \in \mathbb{Z}_{[1, N]}$, as defined in (15). Above, $h_{i, j} \in \mathbb{Z}_{+}$, $i, j \in \mathbb{Z}_{[1, N]}$, is the interconnection delay from the $j$ th to the $i$ th subsystem. It is assumed that $h_{i, i}=0$ for all $i \in \mathbb{Z}_{[1, N]}$. Hence, as it was also the case for (15), for ease of notation, $x_{i}(k)$ appears twice as an argument of $f_{i}$. To describe the complete interconnected system, let $x:=\operatorname{col}\left(\left\{x_{l}\right\}_{l \in \mathbb{Z}_{[1, N]}}\right) \in \mathbb{R}^{n}$, which yields a system

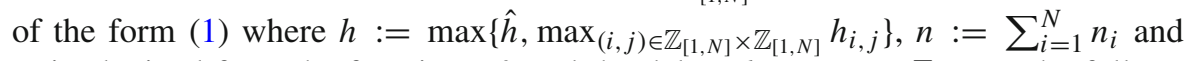
$F$ is obtained from the functions $f_{i}$ and the delays $h_{i, j}, i, j \in \mathbb{Z}_{[1, N]}$. The following corollary, which employs the Razumikhin method, can be obtained directly from Theorems 4 and 5.

Corollary 1 Suppose that the subsystems with local delay (15) satisfy the hypothesis of Theorem 5. Then, the interconnected system (1) obtained from (20) is ISS.

Moreover, a similar result can be obtained, using the Krasovskii approach, from Theorems 4 and 6.

Corollary 2 Suppose that the subsystems with local delay (15) satisfy the hypothesis of Theorem 6. Then, the interconnected system (1) obtained from (20) is ISS.

The above general results provide a framework for ISS analysis of interconnected systems with delay. Moreover, the results for interconnected systems with interconnection delays only or with local delays only are recovered as a particular case, i.e., for $\hat{h}=0$ and for $h_{i, j}=0, i, j \in \mathbb{Z}_{[1, N]}$, respectively. Furthermore, note that Corollaries 1 and 2 reduce the ISS analysis of interconnected systems with both interconnection and local delays to the ISS analysis of interconnected systems with local delays only via a delay-independent small-gain condition. As the ISS analysis of interconnected systems with local delays only is in general less complex, Corollaries 1 and 2 provide a simpler tool to analyse ISS for interconnected systems with delay, when compared to the continuous-time results in, e.g., $[1,28]$. Moreover, Corollary 1 provides a counterpart for discrete-time systems to the results presented in $[8,9]$.

\section{Conclusions}

A scalable method to establish input-to-state stability (ISS) for discrete-time interconnected systems with delays was developed. The fact that a difference equation with delay can be expressed as an interconnection of difference equations without delay is the crux of the proposed framework. Based on this fact and small-gain arguments, it was demonstrated that delays on the interconnection channels do not affect the stability of interconnected systems if a delay-independent small-gain condition holds. Furthermore, also using small-gain arguments, ISS for interconnected systems with 
local delays was established via the Razumikhin and the Krasovskii method, respectively. Via a combination of the results for interconnected systems with interconnection and local delays, respectively, a tractable framework for ISS analysis of large-scale interconnected systems with delay was obtained.

The application of the results in this paper to power systems is particularly interesting as interconnection delays are likely to appear in such systems due to the large geographical regions that power systems span. Moreover, local delays can also arise in power systems, for example, due to the presence of human operators in the local control loop. Another advantage of the proposed approach when applied to power systems is that the effects of the failure of a power line or power plant can be studied by changing the scaling functions $\gamma_{i, j}$. Therefore, the application of the results in this paper to large-scale power systems makes an interesting subject for future research.

Acknowledgments Rob H. Gielen and Mircea Lazar acknowledge the support of the Veni grant number 10230, awarded by the Dutch organizations STW and NWO. Andrew R. Teel acknowledges the support of the Air Force Office of Scientific Research grant FA9550-09-1-0203 and the National Science Foundation grants ECCS-0925637 and CNS-0720842.

Open Access This article is distributed under the terms of the Creative Commons Attribution License which permits any use, distribution, and reproduction in any medium, provided the original author(s) and the source are credited.

\section{Appendix: Proof of Theorem 1}

The following lemma and facts are crucial for the proof of Theorem 1.

Lemma 2 Let $\rho \in \mathscr{K}$ and such that $\rho(s)<s$ for all $s \in \mathbb{R}_{>0}$. Then, $\beta(s, k)=\rho^{k}(s)$ implies that $\beta \in \mathscr{K} \mathscr{L}$.

Fact 1 Let $\alpha_{1}, \alpha_{2} \in \mathscr{K}$ and $\beta \in \mathscr{K} \mathscr{L}$, then

(i) $\alpha(s)=\alpha_{1} \circ \alpha_{2}(s)$ implies that $\alpha \in \mathscr{K}$;

(ii) $\alpha(s)=\max \left\{\alpha_{1}(s), \alpha_{2}(s)\right\}$ implies that $\alpha \in \mathscr{K}$;

(iii) $\alpha(s)=\min \left\{\alpha_{1}(s), \alpha_{2}(s)\right\}$ implies that $\alpha \in \mathscr{K}$;

(iv) $\tilde{\beta}(s)=\alpha_{1} \circ \beta(r, s)$ implies that $\tilde{\beta} \in \mathscr{K} \mathscr{L}$.

Lemma 2 was proven in [17] and Fact 1, which was also reported in [17], can be proven using standard arguments for $\mathscr{K}$ functions, see, e.g., [10]. Next, Theorem 1 is proven.

Proof of Theorem 1 It follows from the small-gain condition (4) that the functions $\gamma_{i, j}$ satisfy the hypothesis of Lemma 1 . Therefore, there exist $\sigma_{i} \in \mathscr{K}_{\infty}, i \in \mathbb{Z}_{[1, N]}$, such that

$$
\max _{j \in \mathbb{Z}_{[1, N]}} \gamma_{i, j} \circ \sigma_{j}\left([y]_{j}\right)<\sigma_{i}\left([y]_{i}\right)
$$


for all $y \in \mathbb{R}_{+}^{N} \backslash\{0\}$ and all $i \in \mathbb{Z}_{[1, N]}$. Let $\mu(s):=\max _{i \in \mathbb{Z}_{[1, N]}} \sigma_{i}^{-1} \circ \mu_{i}(s)$ for all $s \in \mathbb{R}_{+}$and let

$$
\gamma(s):=\max _{i \in \mathbb{Z}_{[1, N]}} \max _{j \in \mathbb{Z}_{[1, N]}} \sigma_{i}^{-1} \circ \gamma_{i, j} \circ \sigma_{j}(s)
$$

It follows from (21) that $\gamma(s)<s$ for all $s \in \mathbb{R}_{>0}$. Furthermore, Fact 1 yields that $\gamma \in \mathscr{K}_{\infty}$ and $\mu \in \mathscr{K}$.

Next, consider the candidate ISS-LF (5) and consider any $(x, u) \in \mathbb{R}^{n} \times \mathbb{R}^{m}$. Then,

$$
\begin{aligned}
W(G(x, u)) & =\max _{i \in \mathbb{Z}_{[1, N]}} \sigma_{i}^{-1} \circ W_{i}\left(g_{i}\left(x_{1}, \ldots, x_{N}, u\right)\right) \\
& \leq \max _{i \in \mathbb{Z}_{[1, N]}} \sigma_{i}^{-1} \circ \max \left\{\max _{j \in \mathbb{Z}_{[1, N]}} \gamma_{i, j} \circ W_{j}\left(x_{j}\right), \mu_{i}(\|u\|)\right\} \\
& \leq \max \left\{\max _{i \in \mathbb{Z}_{[1, N]}} \max _{j \in \mathbb{Z}_{[1, N]}} \sigma_{i}^{-1} \circ \gamma_{i, j} \circ \sigma_{j} \circ \sigma_{j}^{-1} \circ W_{j}\left(x_{j}\right), \mu(\|u\|)\right\} \\
& \leq \max \left\{\gamma \circ \max _{i^{\prime} \in \mathbb{Z}_{[1, N]}} \sigma_{i^{\prime}}^{-1} \circ W_{i^{\prime}}\left(x_{i^{\prime}}\right), \mu(\|u\|)\right\} \leq \max \{\gamma \circ W(x), \mu(\|u\|)\} .
\end{aligned}
$$

Furthermore, the equivalence of norms [14] yields that there exist some $c_{1}, c_{2} \in \mathbb{R}_{>0}$ such that

$$
c_{1} \max _{i \in \mathbb{Z}_{[1, N]}}\left\|x_{i}\right\| \leq\|x\| \leq c_{2} \max _{i \in \mathbb{Z}_{[1, N]}}\left\|x_{i}\right\|
$$

Hence, the $\mathscr{K}_{\infty}$ bounds for the ISS-LFs for the systems (2) yield

$$
\min _{i \in \mathbb{Z}_{[1, N]}} \sigma_{i}^{-1} \circ \alpha_{1, i}\left(c_{2}^{-1}\|x\|\right) \leq W(x) \leq \max _{i \in \mathbb{Z}_{[1, N]}} \sigma_{i}^{-1} \circ \alpha_{2, i}\left(c_{1}^{-1}\|x\|\right)
$$

Therefore, let $\alpha_{1}(s):=\min _{i \in \mathbb{Z}_{[1, N]}} \sigma_{i}^{-1} \circ \alpha_{1, i}\left(c_{2}^{-1} s\right)$ and let $\alpha_{2}(s):=\max _{i \in \mathbb{Z}_{[1, N]}} \sigma_{i}^{-1}$ 。 $\alpha_{2, i}\left(c_{1}^{-1} s\right)$. Then, it follows from Fact 1 that $\alpha_{1}, \alpha_{2} \in \mathscr{K}_{\infty}$ and hence the function $W$ is an ISS-LF for the interconnected system (3), which establishes claim (i).

Applying the inequality $W(G(x, u)) \leq \max \{\gamma \circ W(x), \mu(\|u\|)\}$ recursively and using the bounds $\alpha_{1}$ and $\alpha_{2}$ yields that

$$
\begin{aligned}
& \left\|x\left(k, x(0), \mathbf{u}_{[0, k-1]}\right)\right\| \leq \max \left\{\alpha_{1}^{-1} \circ \gamma^{k} \circ \alpha_{2}(\|x(0)\|), \alpha_{1}^{-1} \circ \mu\left(\left\|\mathbf{u}_{[0, k-1]}\right\|\right)\right\}, \\
& \forall k \in \mathbb{Z}_{\geq 1}
\end{aligned}
$$

for all $x(0) \in \mathbb{R}^{n}$, all $\mathbf{u}_{[0, k-1]}:=\{u(i)\}_{i \in \mathbb{Z}_{[0, k-1]}}$, with $u(i) \in \mathbb{R}^{m}$. Above, $\{x(k, x(0)$, $\left.\left.\mathbf{u}_{[0, k-1]}\right)\right\}_{k \in \mathbb{Z}_{+}}$was used to denote a trajectory of (3) from initial condition $x(0) \in \mathbb{R}^{n}$ with input $\mathbf{u}_{[0, k-1]}$. Letting $\beta(r, s):=\alpha_{1}^{-1} \circ \gamma^{s} \circ \alpha_{2}(r)$ it follows, from the fact that $\gamma(s)<s$ for all $s \in \mathbb{R}_{>0}$, Lemma 2 and Fact 1 , that $\beta \in \mathscr{K} \mathscr{L}$. Therefore, the interconnected system (3) is ISS with $\beta \in \mathscr{K} \mathscr{L}$ and $\gamma_{u} \in \mathscr{K}$, where $\gamma_{u}(s):=\alpha_{1}^{-1} \circ \mu(s)$, which establishes claim (ii) and completes the proof. 


\section{References}

1. Dashkovskiy SN, Naujok L (2010) Lyapunov-Razumikhin and Lyapunov-Krasovskii theorems for interconnected ISS time-delay systems. In: 19th International symposium on mathematical theory of networks and systems, Budapest, Hungary, pp 1179-1184

2. Dashkovskiy SN, Rüffer BS, Wirth FR (2010) Small gain theorems for large scale systems and construction of ISS Lyapunov functions. SIAM J Control Optim 48(6):4089-4118

3. Gielen RH, Lazar M (2011) Stabilization of polytopic delay difference inclusions via the Razumikhin approach. Automatica 47(12):2562-2570

4. Gielen RH, Lazar M, Kolmanovsky IV (2012) Lyapunov methods for time-invariant delay difference inclusions. SIAM J Control Optim 50(1):110-132

5. Gu K, Kharitonov VL, Chen J (2003) Stability of time-delay systems. Birkhäuser, Boston

6. Hetel L, Daafouz J, Iung C (2008) Equivalence between the Lyapunov-Krasovskii functionals approach for discrete delay systems and that of the stability conditions for switched systems. Nonlinear Anal Hybrid Syst 2(3):697-705

7. Ito H, Pepe P, Jiang ZP (2009) Construction of Lyapunov-Krasovskii functionals for interconnection of retarded dynamic and static systems via a small-gain condition. In: Proceedings of the 48th IEEE conference on decision and control, Shanghai, China, pp 1310-1316

8. Ito H, Pepe P, Jiang ZP (2010) A small-gain condition for iISS of interconnected retarded systems based on Lyapunov-Krasovskii functionals. Automatica 46(10):1646-1656

9. Ito H, Jiang ZP, Pepe P (2011) A small-gain methodology for networks of iISS retarded systems based on Lyapunov-Krasovskii functionals. In: Proceedings of the 18th IFAC world congress, Milano, Italy, pp 5100-5105

10. Jiang ZP, Wang Y (2001) Input-to-state stability for discrete-time nonlinear systems. Automatica 37:857-869

11. Jiang ZP, Lin Y, Wang Y (2008) Nonlinear small-gain theorems for discrete-time large-scale systems. In: Proceedings of the 27th Chinese control conference, Kunming, China, pp 704-708

12. Karafyllis I, Jiang ZP (2011) A vector small-gain theorem for general nonlinear control systems. IMA J Math Control Inf 28(3):309-344

13. Kolmanovskii V, Myshkis A (1999) Introduction to the theory and applications of functional differential equations. Kluwer Academic Publishers, Dordrecht

14. Kreyszig E (1989) Introductory functional analysis with applications. Wiley, New York

15. Laila DS, Nešić D (2003) Discrete-time Lyapunov-based small-gain theorem for parameterized interconnected ISS systems. IEEE Trans Autom Control 48(10):1783-1788

16. Lakshmikantham V, Matrosov VM, Sivasundaram S (1991) Vector Lyapunov functions and stability analysis of nonlinear systems. Kluwer Academic Publishers, Dordrecht

17. Limon D, Alamo T, Salas F, Camacho EF (2006) Input to state stability of min-max MPC controllers for nonlinear systems with bounded uncertainties. Automatica 42(5):797-803

18. Liu B, Hill DJ (2009) Input-to-state stability for discrete time-delay systems via the Razumikhin technique. Syst Control Lett 58:567-575

19. Liu B, Marquez HJ (2007) Razumikhin-type stability theorems for discrete delay systems. Automatica 43(7):1219-1225

20. Liu T, Hill DJ, Jiang ZP (2010) Lyapunov formulation of ISS cyclic-small-gain in discrete-time dynamical networks. In: Proceedings of the 8th WCICA, Jinan, China, pp 568-573

21. Michel AN, Miller RK (1977) Qualatitive analysis of large scale dynamical systems, Mathematics in Science and Engineering, vol 134. Academic Press, Inc., New York

22. Orero SO, Irving MR (1998) A genetic algorithm modelling framework and solution technique for short term optimal hydrothermal scheduling. IEEE Trans Power Syst 13(2):501-518

23. Polushin I, Marquez HJ, Tayebi A, Liu PX (2009) A multichannel IOS small gain theorem for systems with multiple time-varying communication delays. IEEE Trans Autom Control 54(2):404-409

24. Raimondo DM, Magni L, Scattolini R (2007) Decentralized MPC of nonlinear systems: an input-tostate stability approach. Int J Robust Nonlinear Control 17:1651-1667

25. Rüffer BS, Sailer R, Wirth FR (2010) Comments on "a multichannel ios small gain theorem for systems with multiple time-varying communication delays". IEEE Trans Autom Control 55(7):1722-1725

26. Teel AR (1996) A nonlinear small gain theorem for the analysis of control systems with saturation. IEEE Trans Autom Control 41(9):1256-1270 
27. Teel AR (1998) Connections between Razumikhin-type theorems and the ISS nonlinear small gain theorem. IEEE Trans Autom Control 43(7):960-964

28. Tiwari S, Wang Y (2010) Razumikhin-type small-gain theorems for large-scale systems with delays. In: Proceedings of the 49th IEEE conference on decision and control, Atlanta, GA, pp 7407-7412

29. Tiwari S, Wang Y, Jiang ZP (2009) A nonlinear small-gain theorem for large-scale time delay systems. In: Proceedings of the 48th IEEE conference on decision and control, Shanghai, China, pp 7204-7209

30. Vidyasagar M (1981) Input-output analysis of large-scale interconnected systems. Lecture notes in control and information sciences, vol 29. Springer, Berlin

31. Šiljak DD (1978) Large-scale dynamic systems: stability and structure. North-Holland, Amsterdam

32. Wang C, Shahidehpour SM (1993) Power generation scheduling for multi-area hydro-thermal systems with tie line constraints, cascaded reservoirs and uncertain data. IEEE Trans Power Syst 8(3):13331340

33. Willems JC (1972) Dissipative dynamical systems. Arch Ration Mech Anal 45:321-393 